\title{
The secular variations of cosmic ray cutoff rigidities, caused by century variations in geomagnetic field, and cosmic ray variations
}

\section{Gvozdevsky Boris}

Polar Geophysical Institute, Firsman Str., 14, Apatity, Russia, 184209; E-mail gvozdevsky@pqia.ru

\section{Dorman Lev ${ }^{1}$}

Israel Cosmic Ray and Space Weather Center Institute of Advanced Study of Tel Aviv University, P.O. Box, 39040, Tel Aviv, ISRAEL, 69978; IZMIRAN; E-mail: I id010529@omai I.com

\section{Belov Anatoly}

Pushkov Institute of Terrestrial Magnetism, Ionosphere and Radio Wave Propagation, Russian Academy of Sciences, IZMIRAN, Moscow, Russia, 108840; E-mail: abeloveizmiran.ru

\section{Gushchina Raisa}

Pushkov Institute of Terrestrial Magnetism, Ionosphere and Radio Wave Propagation, Russian Academy of Sciences, IZMIRAN, Moscow, Russia, 108840; E-mail: rquseizmiran.ru

\section{Eroshenko Eugeniya}

Pushkov Institute of Terrestrial Magnetism, Ionosphere and Radio Wave Propagation, Russian Academy of Sciences, IZMIRAN, Moscow, Russia, 108840; E-mail: erosheizmiran.ru

\section{Preobrazhensky Maxim}

Pushkov Institute of Terrestrial Magnetism, Ionosphere and Radio Wave Propagation, Russian Academy of Sciences, IZMIRAN, Moscow, Russia, 108840; E-mail: planeswalker. ipsumeqmai I. com

\section{Yanke Victor}

Pushkov Institute of Terrestrial Magnetism, Ionosphere and Radio Wave Propagation, Russian Academy of Sciences, IZMIRAN, Moscow, Russia, 108840; E-mail: yanke@izmiran.ru

\begin{abstract}
Vertical rigidities of geomagnetic cutoff are obtained for world neutron monitor network with annual resolution for the period of 1950-2020 by the method of trajectory calculations with the model International Geomagnetic Reference Field. Results of the computations show the total decrease of the cut off rigidities in the majority points which is caused by a decrease of the total geomagnetic field during this period. The prognosis of planetary distribution of the cut off rigidities is made up to 2050 .
\end{abstract}

35th International Cosmic Ray Conference - ICRC2017

10-20 July, 2017

Bexco, Busan, Korea

Speaker L. I. Dorman 


\section{Introduction}

Магнитосферный эффект космических лучей в настоящее время достаточно хорошо изучен. Nevertheless, modern development of the experiment requires more rigorous studies of magnetospheric effects in terms of both the duration of observations and the accuracy of the experimental data. This is associated, for example, with the fact that the geomagnetic field has decreased by about $4 \%$ for the sixty-year period of cosmic ray observations, and it decreases at a different rate in different regions. Impresses that the contribution of high harmonicas of the geomagnetic field for this period, on the contrary, increased by $29 \%$. Besides, magnetic anomalies have the common tendency to a drift to the west (fig.1). Within the 20th century not dipole field drifted to the west with an average speed of $0.15 \%$ year [Wei Zi-Gang et al., 2001].

For practical purposes to estimate consequences of such big reorganization of a magnetic field from the point of view of magnetospheric effects of cosmic rays, it is necessary:

1) To receive planetary distributions and geomagnetic cut off rigidities for network of stations for the entire period of observations;

2) To estimate long-term changes of geomagnetic cut off rigidities of cosmic rays retrospectively and to execute the forecast for the near-term outlook;

3) To estimate the expected century variations of a neutron and muon components of cosmic rays caused by geomagnetic variations.
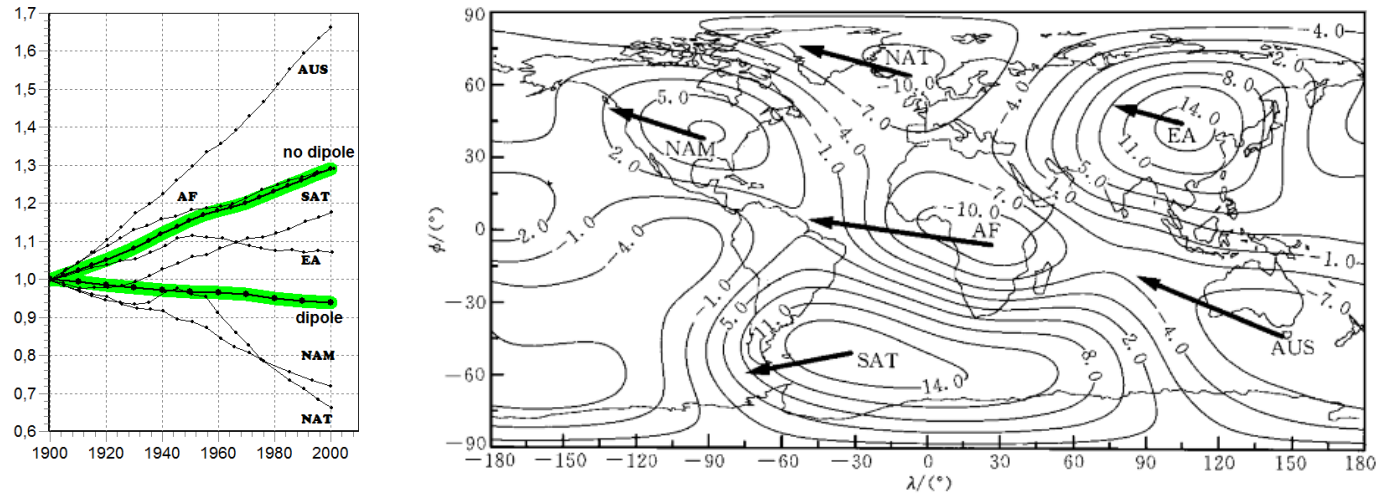

Fig. 1 The relative changes of coefficients of dipole and not dipole terms of expansion of a magnetic field of Earth (at the left) and movement of inhomogeneities during 1900-2000 (on the right). Velocity of movement (in \%year) of six magnetic anomalous [Wei Zi-Gang et al., 2001]:

AF - near Africa (0.26), NAM - in North America (0.12), SAT - near South Atlantic (0.04),

AUS - near Australia (0.23), $\quad$ EA - in Europe and Asia (0.09), NAT - near North Atlantic (0.13).

The magnetospheric effect of cosmic rays is considered as impact on an external stream of space radiation of changes of a condition of a magnetosphere. In an isotropic approximation intensity of cosmic rays $\mathrm{N}$ in point of observation can be presented through primary spectrum of cosmic rays in the form $J(R)$ in the form:

$$
N=\int_{0}^{\infty} \delta(R) \cdot m\left(R, h_{0}\right) J(R) d R=\int_{R_{c}}^{\infty} m\left(R, h_{0}\right) J(R) d R,
$$

where $m\left(R, h_{0}\right)$ is the integral multiplicity of particles of certain type in the Earht's magnetosphere. Whether will overcome or not the charged particle a magnetosphere - reflects in the equation (1) discrete function. If an efficient threshold of the allowed area to designate Rc, then observed variations can be written down, as shown in a right part of expression (1). The characteristic of Rc defined as a rigidity of geomagnetic cut off, completely is determined by a magnetosphere of Earth and allows to define change of an isotropic cosmic ray flux. 
Varying the last expression by all possible parameters (e.g., $R_{c}, m\left(R, h_{0}\right)$ и $\left.J(R)\right)$, for count rate variations of each detector (Indexes omitted) we can write:

$$
\frac{\delta N}{N}=\overbrace{-\delta R_{c} W\left(R_{c}, h_{0}\right)}^{\text {magnetospheric }}+\overbrace{\int_{R_{c}}^{\infty} W\left(R, h_{0}\right) \frac{\delta m}{m}\left(R, h_{0}\right) d R}^{\text {atmospheric }}+\overbrace{\int_{R_{c}}^{\infty} W\left(R, h_{0}\right) \frac{\delta J}{J}(R) d R}^{\text {primary.-variation }},
$$

where items in an isotropic approximation describe three types of variations: magnetosphere, atmospheric and primary variations correspondingly, and $W\left(R, h_{0}\right)$ is the coupling function for the secondary component of the cosmic rays.

An extensive and most comprehensive and systematic investigation of magnetosphere effects of cosmic rays, including long-term changes, were carried out in large series of works by Shea and Smart (1967-2007). Global distributions of the vertical geomagnetic cutoff rigidity were computed for the nine 5-years epochs using trajectory calculations based on the geomagnetic field model. Details and references may be found in [Gvozdevsky et al., 2016].

Shea and Smart (1975b) noticed the uneven distribution of changes in the planetary distribution of geomagnetic cutoff rigidities for 20 years from 1955 to 1975 , especially in the northern and southern parts of the Atlantic Ocean. In the southern part of the Atlantic Ocean, a decrease in rigidity was observed, while a comparable increase in the vertical cutoff rigidity occurred in the northern part.

Such calculations were not conducted for the existing network of detectors over the past four epochs. In addition, over the past 20 years, about a third of the new neutron monitors were added to the system and a network of multidirectional muon telescopes was created; the geomagnetic cutoff rigidity and asymptotic reception cones for these detectors should be computed for the first time.

\section{Method of calculation}

Currently, the generally accepted and the most accurate way to determine the geomagnetic cutoff rigidity is the method of trajectory calculations based on the solution of the equation of motion of charged particles in the geomagnetic field. The equation of motion was solved numerically by the Runge-Kutta method on the fourth order of accuracy with adaptive step.

The particles start from a height of $20 \mathrm{~km}$. Integration is completed in three cases: either a particle penetrates to a depth of less than $\left(R_{E}+20\right) \mathrm{km}\left(R_{E}\right.$ is the radius of the Earth) (the particle returns back into the atmosphere, trajectory 0 , Fig. 1), the integration is completed at the end of the target time (the particle is considered captured, trajectory 1), or the particle crosses the surface of the magnetopause (moves beyond the magnetosphere, trajectories 2-5). In the latter case, the trajectory is defined as allowed, otherwise, as forbidden. As a result of the calculation, a discrete penumbra function $g(R)$ is formed; it takes values " 0 " or " 1 " for all rigidity values with the selected step of $0.001 \mathrm{GV}$.

The model of the main magnetic field IGRF is involved starting from 1900 up to 2015 with 5-year interval. Taking into account secular variations of the magnetic field, the model is continued until 2020 (IGRF-12 model, 2015). The field is represented by ten or 13 spherical harmonics, but we limited us to ten harmonics for the entire period and to eight harmonics for the predictive model of the field

In the last decade the soft ware complex PLANETOCOSMICS Geant4 v6 [Desorgher, 2006] is used for such tasks, on which base the following are performed: [Zreda, 2012; Herbst et al., 2013; Boschini et al., 2014; Doetinchem, 2017]. Unfortunately PLANETOCOSMICS is not supported last time.

\section{Discussion of the results. Planetary distribution of the rigidities of geomagnetic cutoff.}

Before proceeding to the calculation of the geomagnetic cutoff rigidity for the network of stations of neutron monitors, the planetary distributions of geomagnetic cutoff rigidities were calculated for the IGRF for all epochs from 1950 to 2020 with a step of five years for the grid $5^{\circ}$ 
$\times 15^{\circ}$ in latitude and longitude. Digital and graphical results can be found on the website [Table of planetary distributions $\left.R_{c}, 2016\right]$. An example of such presentation for the epoch 2015.0 is shown in Figure 2 (in gradient representation - at the left and in the form of isolines of rigidities of geomagnetic cutoff - on the right)
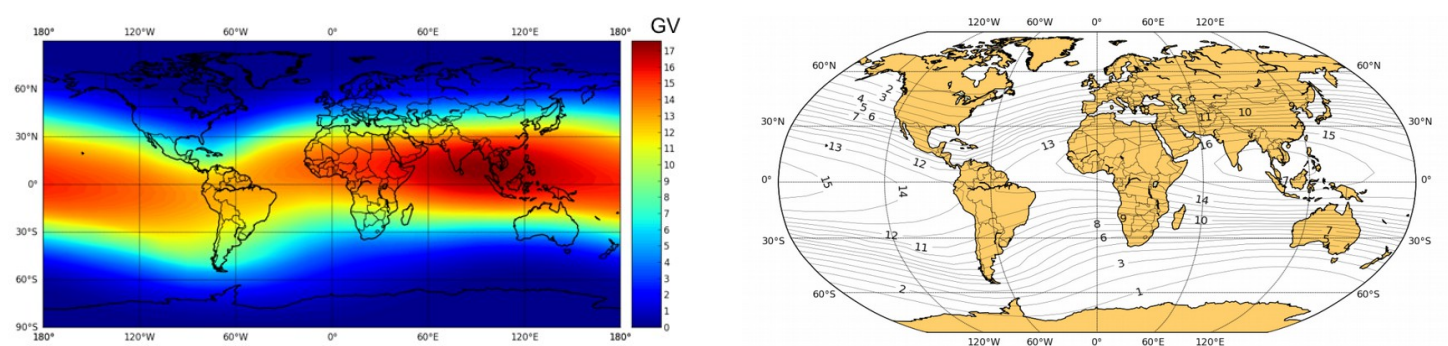

Fig. 2. Planetary distribution of the vertical rigidities of geomagnetic cutoff for the epoch 2015.0 (in GV for gradients or isoline values).

For obtaining dynamics of changes of rigidities of geomagnetic cutoff changes of planetary distribution of rigidities relatively to basic epoch were defined (for example, 1950.0). One of results is given in fig.3. The most strong bright areas of changes are in northern and

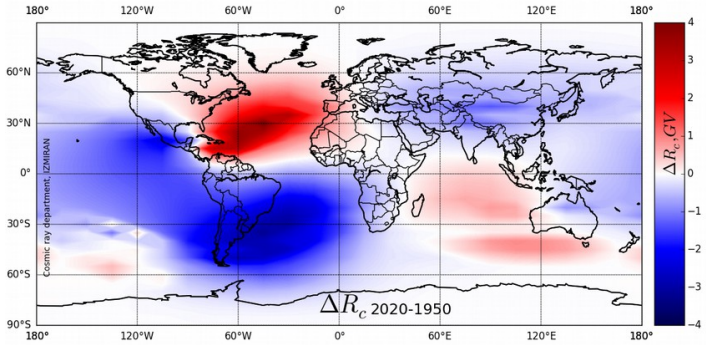

Fig. 3. Changes of the planetary distribution of the vertical cutoff rigidity relatively to epoch 1950 [Animation_2020-1950, 2015]. southern Atlantic. But huge east loop Europe Africa the Indian Ocean Australia is observed in the first case, in the second case a huge, but the western loop - the Silent ocean - Asia is formed.

Also other graphic representations [Animation 2050-1950, 2016; Animation 2050-1900, 2017] which allow visualizing dynamics of changes of geomagnetic cutoff rigidities from the past in the future are executed.

\section{Discussion of the results. The rigidities of geomagnetic cutoff for world network of the stations.}

Common imagine on the changes of the geomagnetic cutoff rigidities of the global network of cosmic ray stations are given in Fig. 4, the results of which were obtained for the IGRF model. Computations were carried out for 124 stations with one year resolution for the entire period from 1950 to 2020 (for 2020 the prognostic model was used). The figure shows the changes in geomagnetic cutoff rigidities with respect to the epoch 1950 for the entire period of continuous monitoring of cosmic rays for four groups of CR stations. The temporal changes of geomagnetic cutoff rigidities of high-latitude detectors $(<3 \mathrm{GV})$ reach $0.5 \mathrm{GV}$, with different signs. For the American, Canadian, and European stations, a systematic increase of cutoff rigidities is observed. This is because of the influence of the peripheral abnormal region in the northern part of the Atlantic, as seen in Fig. 4. For other detectors, there is a slight decrease of cutoff rigidities. For intermediate values in the range of 3-8 GV, geomagnetic cutoff rigidities generally decrease, with the exception of several detectors of the western coast of Europe, an area that is the periphery of the North Atlantic anomaly. In low-latitude regions up to the equatorial, a significant decrease in geomagnetic cutoff rigidities is also observed for all detectors. Thus, there is a global decrease in geomagnetic cutoff rigidities with the exception of the North Atlantic anomaly. For low-latitude detectors, up to equatorial, essential decrease in geomagnetic cutoff rigidities is also observed. Thus, global decrease in rigidities is observed, except for North Atlantic anomaly where, unfortunately, there are no operating detectors, and slight increase near Australia exists. 

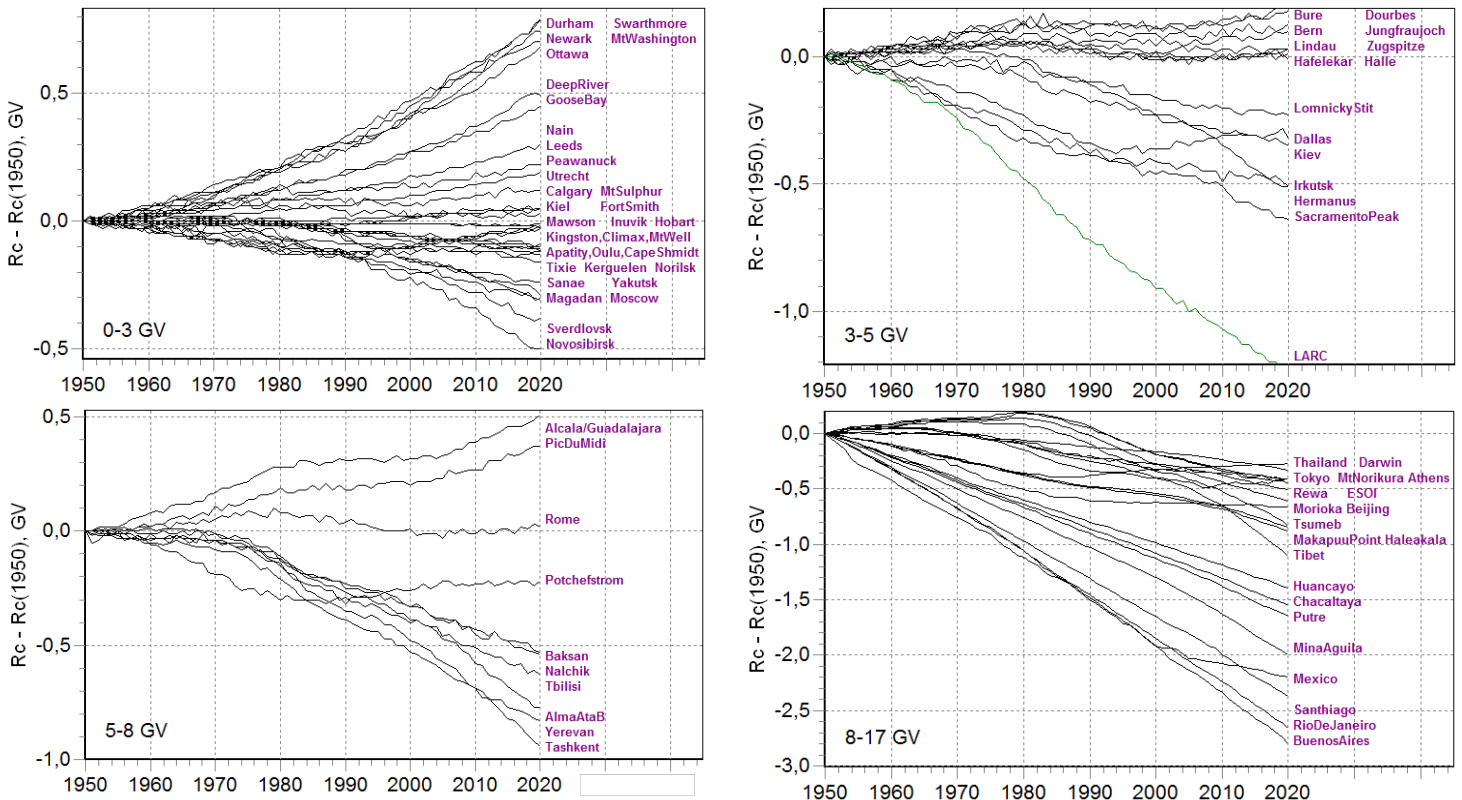

Fig. 4Relative change of vertical geomagnetic cutoff rigidities for 4 groups of CR stations with respect to epoch 1950 (with IGRF model of field and flat spectrum of CR variations).

The results for all detectors one can find on the resource [Local value tables $\mathrm{R}_{\mathrm{c}}, 2016$ ].

\section{Discussion of the results. Contribution of the external magnetic field in long term changes of the threshold}

The Earth's magnetosphere is exposed to long-term changes caused by solar activity. In order to estimate these changes, the Tsyganenko model of the magnetic field can be used, for example, Ts89 (Tsyganenko, 1989). The input parameter of this model is the $K p$-index. Since the $K p$-index is the logarithmic index, annual average values of the $A p$-index were used in order to obtain annual averages of the $K p$-index after the corresponding recalculation.

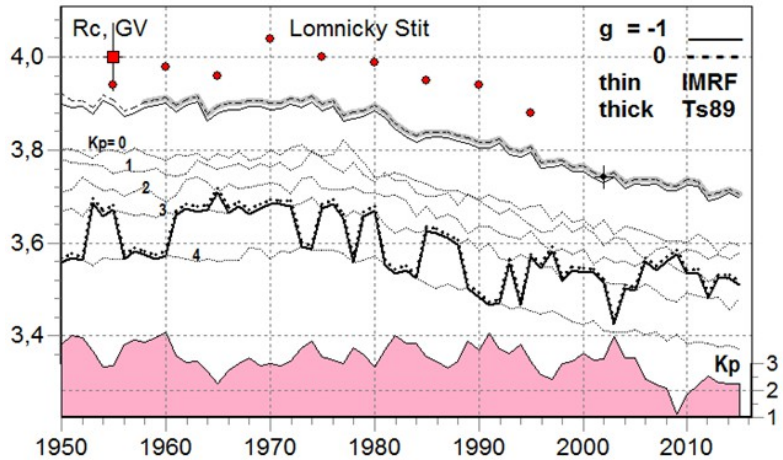

Fig. 5. Changes of the cutoff rigidity for Lomnicky Stit at two models of magnetosphere (IMRF and Ts89) with accounting penumbra under flat $(\gamma=0)$ and power $(\gamma=1)$ spectra of the CR variations.

For example in Figure 5 the result for mid-latitude station Lomnicky Stit is presented. The top couple of curves falls into calculations for the IMRF model and the accounting of a penumbra in an approximation flat (at $\gamma=0$, dotted curve) and a power spectrum Estimation of an accuracy for the epoch 2002 gives $\sim 0.05 \mathrm{GV}$.

Also comparison with results of the first trajectory calculations is given in fig.5 [Shea et al., 1965], received for expansion of a magnetic field on the first six harmonicas for an era of 1955 (a square, with accuracy $0,1 \mathrm{GV}$ ).

The circles show the result also obtained by a method of trajectory calculations but for the field IGRF [Shea at al., 2001] for all the stations of world wide network. These computations give on $0.1 \mathrm{GV}$ less rigidity that we connect it with more exact step $(0.01 \mathrm{GV})$ of integration of the equation of moving.

Rigidities of geomagnetic cutoff obtained with Tsyganenko model (Ts89) are depicted in the middle part of Fig. 5 for different values of $\mathrm{Kp}$ index (from 0 to 4). The values of the $\mathrm{Kp}$ - 
index show the possible corridor of changes of geomagnetic cutoff rigidities, within which the actual values change depending on the value of the Kp index. The lower part of Fig. 5 shows the average value of the $K p$-index. For the period from 2015 to 2020 marked by the bold line in the lower part of Fig. 5, we used predicted values of the $K p$-index.

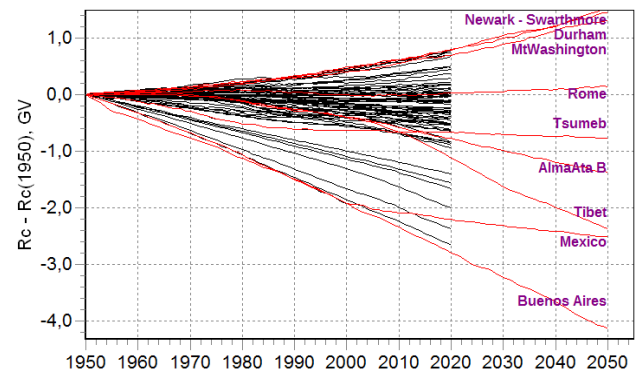

Fig. 6. Prognosis of the changes of vertical geomagnetic cut off rigidity relatively to epoch of 1950 for some stations up to 2050

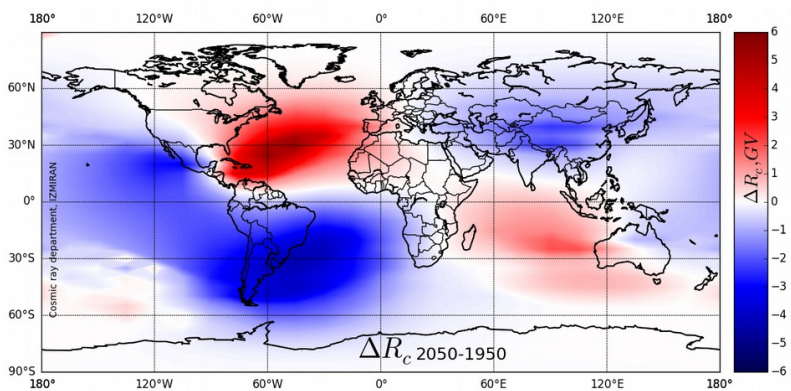

Fig. 7. Prognosis up to 2050 of the changes of planetary distribution of vertical geomagnetic cut off rigidity relatively to epoch 1950 (Animation 2050-1950), and to epoch 1900 (Animation 2050-1900).

The calculation shows that an accounting for the influence of the outer magnetosphere on long-term changes of cutoff rigidities reduces geomagnetic cutoff rigidities by a few tenths of GV and reaches about $0.3 \mathrm{GV}$ for high-latitude detectors, for example, Kerguelen, Deep River, Durham, and Apatity stations. All observed changes in the cutoff rigidity are in opposition to the changes of the $K p$-index. For mid-latitude stations, this influence is reducesed (Fig. 10) and vanishes for low-latitude detectors. The results for all detectors of worldwide network can be found on resource [Graphical representation $R_{c}, 2017$ ].

\section{Discussion of the results. Prediction of the changes of geomagnetic cutoff rigidities.}

The forecast of vertical geomagnetic cut off rigidity with respect to an era of 1950 is

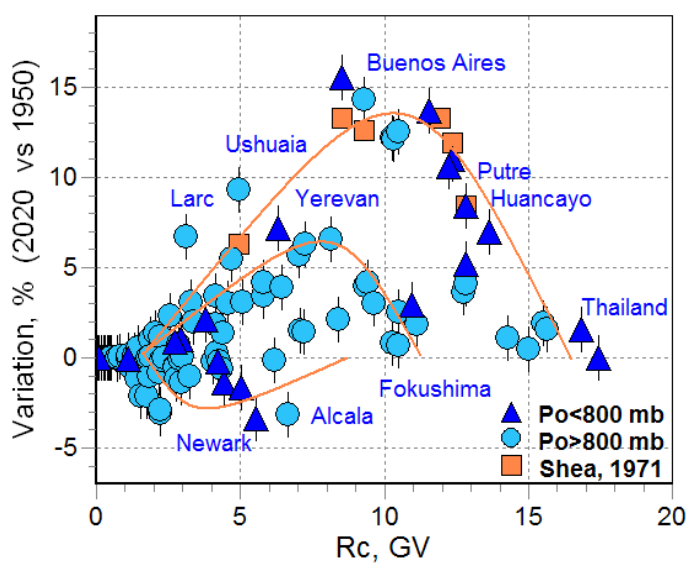

Fig. 8. The expected century variations of the neutron component for World network of CR stations caused by changes of the geomagnetic field. Triangles mean mountain stations provided in approach of linear extrapolation of coefficients of decomposition of magnetic field of all the harmonicas for eras 2020.0 2050.0 on fig. 6 for some selected stations. In the accepted approach abnormal development of behavior of geomagnetic cut off rigidity continues though it is impossible to claim with reliability that such linearity will be true. Planetary distribution of geomagnetic cut off rigidity for future eras is given in fig.7. Such forecast can be useful at design of long-term experiments.

\section{Discussion of the results. Centure cosmic ray variations caused by the changes of geomagnetic cutoff rigidities.}

Planetary changes of threshold cutoff rigidities have to lead to the corresponding variations of $\mathrm{CR}$ intensity of a magnetosphere origin. First composed the equations (2) describes the corresponding variations which are defined only by changes of geomagnetic cut off rigidity relatively to basic period and coupling function in a point of observation. For stations of World network the expected variations for 2020 relatively 1950 are shown in fig. 8 .

The expected variations can conditionally be divided into two groups. The stations which are in near and peripheral zones of the southern Atlantic anomaly for which reduction of 
geomagnetic cut off rigidity and, respectively, positive variations are observed concern the first group (fig.8, the top and average group of points). Stations which are in a peripheral zone of North Atlantic anomaly for which increase in geomagnetic cut off rigidity and, respectively, negative variations of cosmic rays are observed concern the second group (fig.8, the lower group of points). These are regions of Europe and the Indian Ocean.

Also the results of work [Shea, 1971] received for six South American stations which are shown by small squares in the sequence are given in fig.8: Ushuaia, Mexico City, Buenos Aires, Mina Aguilar, Chacaltaya, Huancayo. The expected variations of a magnetosphere origin have been received from the latitudinal servey. The good example of the same approach is given in work [Herbst et al., 2013] in which the expected effect has been estimated from the latitudinal curve in the period of solar activity minimum for two couples of stations: Moscow- Kiel and Mexico City- British Virgin Island stations (a hypothetical point, $18^{\circ} \mathrm{N}, 64{ }^{\circ} \mathrm{W}$ ). Planetary distribution of the expected CR variations of a magnetosphere origin for 2020 relatively 1950 are shown on fig.9 (in gradient representation - at the left and in the form of isolines of the expected variations - on the right).
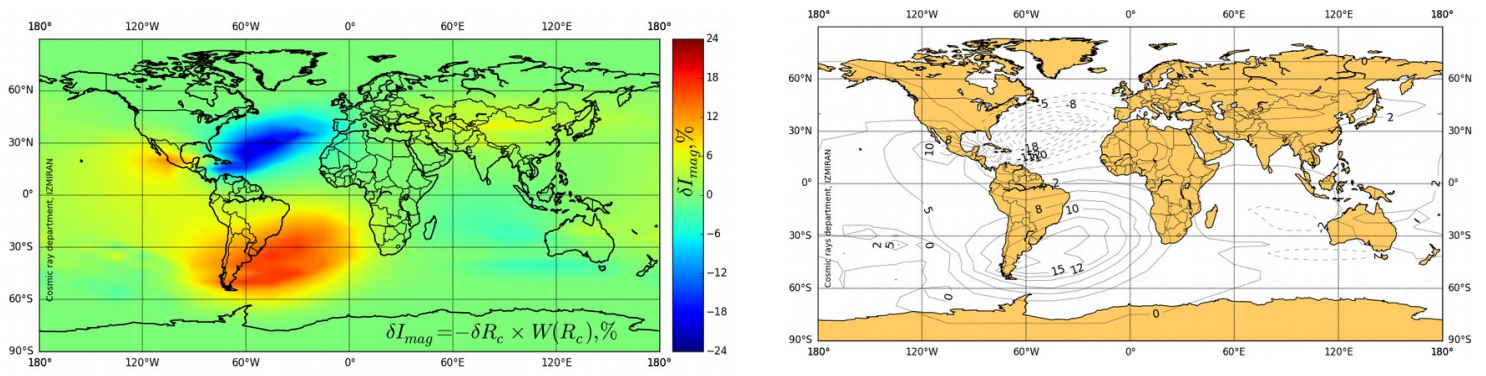

Fig. 9. Planetary distribution of the expected century cosmic ray variations of magnetosphere origin (in \% for gradient and values of isolines)

\section{Conclusions}

The reason for the significant change in geomagnetic cutoff rigidities in 1955-2020 is a general decrease in the magnetic field of the Earth, against the background of which a certain "contrast" increases with the presence of regions with a sharp change in the field near the poles and magnetic anomalies, as well as changes in the displacement vector of the poles and anomalies.

This leads to the long term changes in the effective geomagnetic cut off rigidities for the world wide neutron monitor network. For the stations which are under the influence of North Atlantic anomaly the negative magnetospheric effects are watched, anomaly drifts to the west with a speed of $0.14 \%$ year. For the stations which are under the influence of the southern Atlantic anomaly the positive magnetosphere variations are observed, and amplitude of such variations reaches $10 \%$, anomaly drifts to the west with a speed of $0.30^{\circ} /$ year. Such effect needs to be considered at an assessment of a spectrum of long-term cosmic ray variations. All results of calculations are available in a digital or graphic form on a resource [Magnetospheric_Effect, 2015].

\section{Acknowledgments}

This work was partially supported by the Program of the Presidium of RAS № 23 "High energy physics and neutrino astrophysics", RFBR grant № 17-02-00508, experimentally and methodologically support the project UNU №85 "Russian national network of ground stations of cosmic rays" We are grateful to all the staff of the World Network of cosmic ray stations http://cr0.izmiran.ru/ThankYou and to http://nmdb.eu. 


\section{References}

Magnetospheric_Effect ftp://crsb.izmiran.ru/Magnetospheric Effect. ePub, 2015.

Animation ftp://crsb.izmiran.ru/Magnetospheric Effect/GRID Delta 1950/Plot 2050-1950 (Planetary distribution of $\mathrm{R}_{\mathrm{c}}$ relative to the 1950 era). ePub, 2016.

Animation ftp://crsb.izmiran.ru/Magnetospheric_Effect/GRID_Delta 1900/Plot_2050-1900 (Planetary distribution of $\mathrm{R}_{\mathrm{c}}$ relative to the 1900 era). ePub, 2017.

Animation ftp://crsb.izmiran.ru/Magnetospheric_Effect/VariationCR_FromRcChange/Animation_mag_variatio n_CR.gif (Dependence of cosmic ray variations due to secular changes $R_{c}$ ). ePub, 2017.

Graphical representations of $R_{c}$ for the network of stations for 1950-2020. ePub, 2017. $\mathrm{ftp} / / / \mathrm{crsb}$.izmiran.ru/Magnetospheric_Effect/AnimationStationRc-2s.gif

Table of planetary distributions of Rc for 1900-2020. ePub, 2016. ftp://crsb.izmiran.ru/Magnetospheric Effect/GRID 1900-2050 Rc

Table values of $\mathrm{Rc}$ for the network of stations for 1950-2020. ePub, 2016. ftp://crsb.izmiran.ru/Magnetospheric Effect/Data IGRF/

Model IGRF-12. ePub. http://www.ngdc.noaa.gov/IAGA/vmod/igrf.html. 2015.

Gvozdevsky B.B., Abunin A.A., Kobelev P.G., Gushchina R.T., Belov A.V., Eroshenko E.A., Yanke V.G. "Magnetospheric Effects of Cosmic Rays. I. Long-Term Changes in the Geomagnetic Cutoff Rigidities for the Stations of the Global Network of Neutron Monitors" ISSN 0016-7932, Geomagnetism and Aeronomy, 2016, Vol. 56, No. 4, pp. 381-392. 2016. doi:10.1134/S0016793216040046. |슬 pdf | |슬 pdf $\mid$

Doetinchem P., Yamashiro Bryan, "Geomagnetic Cutoff Calculations for the Interpretation of Lowrigidity Cosmic-ray Antiparticle Measurements", JGR, in press, doi:10.1002/, 2017|씨 pdf|

Zreda Marek. COSMOS project University of Arizona. Cutoff rigidity online Calculator, model IGRF. Epub. 2012. http://cosmos.hwr.arizona.edu/Util/rigidity.php

Boschini M.J., Della T.S., Gervasi M., Grandi D., Rancoita P. G. ,Bobik P., Kudela K. // Cutoff rigidity online Calculator, model T96, T05. E-pub. 2014. http://www.geomagsphere.org/geomag

Shea M.A., Smart D.F., McCracken K.G. A study of vertical cutoff rigidities using sixth degree simulations of the geomagnetic field // J. Geophys. Res. V.70. P. 4117-4130. doi: 10.1029/JZ070i017p04117. 1965. | |s pdf

Shea M.A., Smart D.F. A five by fifteen degree world grid of calculated cosmic ray vertical cutoff rigidities for 1965 and 1975 // Proc. 14th ICRC, Munchen, V.4. P. 1298-1303. 1975. |l pdf|

Shea M.A., Smart D.F. Vertical cutoff rigidities for cosmic ray stations since 1955 // Proc. 27th ICRC. P. 4063 - 4066. 2001. |스. pdf

Shea M.A. "Changes in neutron monitor response and vertical cutoff rigidities resulting from secular variation in the geomagnetic field" // Proc. 12th ICRC, Hobart, V.3. P. 859-864. 1971. |씨 pdf

Herbst K., Kopp A., Heber B. "Influence of the terrestrial magnetic field geometry on the cutoff rigidity of cosmic ray particles", Annales Geophysicae, 31, 1637-1643, 2013. |시 pdf|

Desorgher, L.: The PLANETOCOSMICS code, (last access: 26 September 2013), 2006, available at: http://cosray.unibe.ch/ laurent/planetocosmics

Wei Zi-Gang, Xu Wen-Yao, "Drifts and intensity variations of the geomagnetic field", Chinese Journal of Geophysics, http://onlinelibrary.wiley.com/doi/10.1002/cjg2.167/epdf, Vol.44, No.4, 496-505, 2001.

Cooke, D. J., Humble, J. E., Shea, M. A., Smart, D. F., and Lund, N.: "On cosmic-ray cut-off terminology", Nuovo Cimento C Geophys. Space Phys. C, 14, 213-234, 1991. 